triphosphatase. Am J Physiol 1986; 251: F603.

14. Maessen JG, van der Vusse GJ, Vork M, Kootstra G. Inability to maintain adenine nucleotide levels by cold storage in ischemically damaged and control kidneys. Transplant Proc 1987; 19: 4112.

15. Pegg DE. Principles of tissue preservation. In: Progress in transplantation, vol 2. Morris PJ, Tilney NL, eds. Edinburgh: Churchill Livingstone, 1985.

16. Rice MJ, Southard JH, Hoffmann RM, Belzer FO. Effects of hypothermic kidney preservation on the isolated perfused kidney: a comparison of reperfusion methods. Cryobiology 1985; 22: 161.
17. Kruuv J, Glofcheski D, Cheng KH, et al. Factors influencing survival and growth of mammalian cells exposed to hypothermia: I. Effects of temperature and membrane lipid perturbers. J Cell Physiol 1983; 115: 179.

18. Hochachka PW. Defense strategies against hypoxia and hypothermia. Science 1986; 231: 234.

Received 10 May 1988.

Accepted 10 July 1988.
$0041-1337 / 89 / 4703-0414 \$ 02.00 / 0$

TRANSPLANTATION

Copyright 1989 by The Williams \& Wilkins Co.
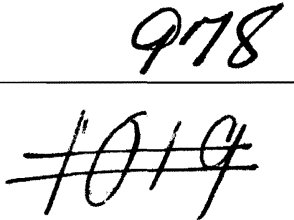

Vol. 47, 414-419, No. 3, March 1989 Printed in U.S.A

\title{
CARDIAC TRANSPLANTATION IN THE RAT
}

\section{The Effect of Histocompatibility Differences on Graft ARteriosclerosis ${ }^{1}$}

\author{
Donald V. Cramer ${ }^{2,3}$ Shiquang Qian, ${ }^{4}$ Jo Harnaha, ${ }^{2}$ Frances A. Chapman, ${ }^{4}$ \\ LarRy W. Estes, ${ }^{2}$ Thomas E. STARzL, ${ }^{4}$ and LeONARD MaKowKa ${ }^{4}$
}

Departments of Pathology and Surgery, University of Pittsburgh School of Medicine, Pittsburgh, Pennsylvania 15261

The development of arteriosclerosis is the most serious and common complication in long-term survivors of cardiac transplantation. We have used a variety of inbred rat strains with selected histocompatibility differences to examine the influence of prolonged, mild rejection reactions on the development of pathological changes in long-term cardiac allografts. Heterotopic cardiac allografts were exchanged between rat strains that differed for MHC class I (RT1.A and/or RT1.E) antigens or groups of minor, non-MHC antigens in MHC-compatible congenic combinations. Our results demonstrate that in strain combinations in which the allograft reaction is mild and prolonged, the donor hearts exhibit pathological changes that include a diffuse, interstitial myocardial fibrosis, perivascular fibrosis, and intimal proliferation in arteries of the graft myocardium. The lesions were less prominent in animals with more active rejection and infrequent in strains that differ for class $I$ histocompatibility antigens or syngeneic controls. These results suggest that the comparable pathological changes seen in long-term human cardiac survivors may reflect low-level, persistent allograft reactions rather than factors associated with graft anoxia or effects of immunotherapy to prevent graft rejection.

Cardiac transplantation has become an important and effective therapeutic alternative for patients with terminal heart disease. The inclusion of cyclosporine as part of the immuno-

\footnotetext{
${ }^{1}$ This work was supported by Grants CA 38804 and AM 29961 from the National Institutes of Health.

${ }^{2}$ Department of Pathology.

${ }^{3}$ Address correspondence to: Dr. Donald V. Cramer, 712 Scaife Hall, University of Pittsburgh School of Medicine, Pittsburgh, PA 15261.

${ }^{4}$ Department of Surgery.
}

suppressive regimen for patients following cardiac transplantation has resulted in a reduction in the severity of rejection episodes and infectious complications, and has improved patient survival to greater than $80 \%$ in the first year after transplantation (1). With the prolonged survival of cardiac allografts, however, has come the recognition of important graftrelated complications in long-term survivors.

The most frequent and important complication of cardiac allografts for patients who survive the first postoperative year is the appearance of an accelerated arteriosclerosis in the coronary arteries of the donor heart (2). The arteriosclerosis consists of a concentric proliferation of the intima that gradually reduces peripheral blood flow and frequently produces myocardial infarction secondary to occlusion of the vessels. At five years after grafting the incidence of graft arteriosclerosis is approximately $50 \%$.

The appearance of similar vascular lesions has been reported in experimental animals after cardiac allografting. In the dog, proliferative intimal lesions were observed in the coronary arteries of orthotopic cardiac allografts that survived for greater than three months $(3)$, suggesting that the lesions were due to chronic rejection of the graft following treatment of the recipients with immunosuppressive agents. Similar vascular lesions have been observed in rabbits $(4,5)$ and in xenografts between subhuman primates (6).

Rat heterotopic cardiac allografts have been shown to develop vascular intimal proliferation if the grafts survive for sufficient periods $(7,8)$. Cardiac grafts exchanged between strains of rats compatible for their class I (RT1.A) histocompatibility antigens developed proliferation of collagen-producing myointimal cells after graft survival of at least 30 days (7). The donor/recipient strain combinations used for these studies presumably differed 
or minor non-MHC histocompatibility loci and perhaps other foci within the MHC, as evidenced by brisk first-set skin graft rjection (10-15 days) and variable cardiac rejection times (9110 days). Reduction in the severity of the allograft reaction by treatment of the recipients with azathioprine $(12 \mathrm{mg} / \mathrm{kg} /$ day) was associated with a concomitant reduction in the degree of intimal proliferation (4). Similar results were obtained with heterotopic cardiac grafts exchanged between ACI donors and $(\mathrm{LEW} \times \mathrm{BN}) \mathrm{F}_{1}$ recipients $(8)$. This donor/recipient strain combination is mismatched for MHC and non-MHC histocompatibility antigens, and grafts that survived 20 to 50 days developed arteriosclerosis. The number and severity of the arterial lesions increased when the rejection process was suppressed by treatment of the recipients with CsA. The degree of vascular involvement did not appear to be related to the severity of the rejection. Previous reports of the toxic effects of CsA on graft arteries ( 9 , 10) and the appearance of vascular lesions in the isograft controls, however, complicated the interpretation of the data.

We have recently conducted a series of experiments in which heterotopic cardiac grafts were exchanged between strains of rats that differ for defined $\mathrm{MHC}$ and non-MHC histocompatibility differences. We have previously demonstrated that incompatibility for selected class I MHC and non-MHC histocompatibility loci may be associated with long-term survival of cardiac $(11,12)$, bone marrow (13), and liver (14) allografts. The donor/recipient combinations used in these studies were selected for allogeneic differences that would provide for prolonged cardiac allograft survival in the absence of immunosuppression, and provide the opportunity to establish the importance of histocompatibility differences on the pathogenesis of the cardiac lesions. Our results demonstrate that prolonged survival of cardiac grafts between donor and recipient combinations with weak allogeneic incompatibilities, are associated with the development of arteriosclerosis, diffuse myocardial fibrosis, and perivascular fibrosis.

\section{MATERIALS AND METHODS}

Animals. Adult rats (10-16 weeks of age), bred in our colony at the University of Pittsburgh School of Medicine, were used in this study. The strains of rats and their genetic characterization are presented in Table 1 . The rats were housed under conventional conditions and fed rat chow (Wayne Lab-Blox F-6, Chicago, IL), and tap water ad libitum.

Heterotopic cardiac grafts. Intraabdominal heterotopic cardiac grafting was performed using a modification of the techniques described by Ono and Lindsey (15). The donor animals were anesthetized with sodium pentobarbital $(40 \mathrm{mg} / \mathrm{kg}$ intraperitoneally) and maintained on methoxyflurane via inhalation. The vena cavae and pulmonary veins were ligated with 5-0 silk, and the pulmonary artery and aorta transected 2-3 mm above their origins in the heart. After perfusion of the ventricles and atria with lactated Ringer's solution (containing $200 \mathrm{U} /$ $\mathrm{ml}$ of heparin), the heart was placed in a saline bath at $4^{\circ} \mathrm{C}$. Recipient animals were anesthetized with pentobarbital, a midline incision made, and the great abdominal vessels dissected free from the left renal vein to the bifurcation. The graft was implanted in the abdominal cavity with both anastomoses done in a running end-to-side fashion with 100 Novafil on a TE-10 needle. Operative times ranged from $30-45 \mathrm{~min}$, with a success rate of approximately $90 \%$. The grafts were evaluated for function by abdominal palpation and all grafts were removed 90 days postoperatively. At removal, the grafts were perfused with $2 \%$ glutaraldehyde in sodium cacodylate buffer $(0.125 \mathrm{M} \mathrm{Na}$ cacodylate, $0.75 \mathrm{M}$ sucrose at $\mathrm{pH} 7.5$ ). The hearts were fixed in solution for $24 \mathrm{hr}$ and then stored in buffer prior to processing.

Histology. All grafts were examined histologically following sectioning and staining with hematoxylin and eosin, Masson's trichrome, and
TABLE 1. Genetic incompatibilities for donor and recipient cardiac allografts

\begin{tabular}{lllcc}
\hline & & & \multicolumn{2}{c}{ Disparity } \\
\cline { 4 - 5 } Group & \multicolumn{1}{c}{ Donor } & Recipient & RT1 & $\begin{array}{c}\text { Non-RT1 (donor strain } \\
\text { non-MHC allogeneic } \\
\text { differences) }\end{array}$ \\
\hline 1 & DA & DA & - & - \\
2 & DA & DA.1I(BI) & RT1.A $^{*}$ & - \\
3 & DA.1I(BI) & (DAXBN)F 1 & RT1.E $^{\mathrm{u}}$ & - \\
4 & DA.1I(BI) & DA & RT1.A $^{n}+E^{u}$ & - \\
5 & DA.1N(BN) & BN & - & DA \\
6 & BN & DA.1N(BN) & - & BN \\
7 & BN.1A(DA) & DA & - & BN
\end{tabular}

Use some other symbol. Small letter designations imply an RT1 haplotype designation. (i.e. RT1*, $\mathrm{RT}^{\mathrm{b}}$, etc.)

Berhoff's elastic stains. Selected specimens were embedded in eponaraldite, and $1-\mu \mathrm{m}$ sections used for examination with the light microscope. Ultrathin sections were stained with uranyl acetate and lead citrate and examined with a Phillips 300 transmission electron microscope. Histologic findings were scored blindly by one pathologist for mononuclear inflammatory infiltrates of the myocardium, perivascular inflammation, and fibrosis and arteriosclerosis of myocardial vessels. All lesions were graded for severity using a scale of 0 (none), $1+$ (trace), $2+$ (mild), $3+$ (moderate), and $4+$ (severe). The severity of the arteriosclerosis lesions was classified by the extent of the lesions and represented changes that were trace-to-mild in one vessel $(1+)$, moderate in one or two vessels $(2+)$, mild-to-moderate in several vessels $(3+)$, or severe in several vessels $(4+)$.

\section{RESULTS}

Graft survival. The genetic incompatibilities for the recipient/donor strain combinations are presented in Table 1. Each of these combinations represents allogeneic differences that do not stimulate acute graft rejection, and all of the hearts included in this study were viable at 90 days posttransplantation. These survival times are in general agreement with the experimental results of cardiac allografting from a variety of laboratories (16). Incompatibility for MHC class I antigens in the rat is a weak stimulus for rejection, and survival times are variable and usually prolonged. In these experiments, the strains selected for grafting expressed RT1.A and/or RT1.E class I histocompatibility antigens that did not stimulate acute rejection of the donor hearts.

Histopathologic findings. General: The pathologic changes observed in the cardiac allografts ranged from none to lesions consistent with prolonged, moderate allograft rejection. In general, the syngeneic controls and strain combinations with MHC class I incompatibilities displayed minimal changes, while the MHC compatible, non-MHC incompatible combinations had lesions consistent with mild-to-moderately severe allograft rejection (Table 2). Two types of histopathologic changes were seen in both control and test animals that were not clearly related to the allograft reaction. The first was the presence of focal inflammatory infiltrates and areas of fibrosis in the subendothelial and epicardial surfaces of the graft (Table 2). The intensity of these reactions was similar between groups and they were considered to represent tissue reactions to the grafting procedure. The second type of pathologic change seen in both the control and test groups was the appearance of focal, transmural areas of myocardial atrophy and fibrosis in the left and/or right ventricles. These lesions were interpreted to represent areas of infarction with subsequent healing and fibrosis. 
The evaluation of the vascular and myocardial lesions associated with the allograft reaction excluded areas containing either type of these two lesions. Two animals, one in group 4 and one in group 6 , had extensive areas of infarction and were excluded from the study.

Syngeneic controls: The pathologic changes seen in the syngeneic grafts exchanged between DA strain animals consisted of mild-to-moderate focal inflammatory lesions with fibrosis in the epicardium and in subendothelial locations in the left and right ventricles. These lesions were consistently present in all the control and test groups (Table 2) and were considered to represent changes secondary to the grafting procedures. None of the control animals exhibited histologic evidence of myocardial or perivascular fibrosis or diffuse inflammation of the myocardium. One animal had focal areas of infarction in both the left and right ventricle.

Class I MHC incompatibilities: Three of the donor/recipient strain combinations differ for either RT1.A and/or RT1.E class I histocompatibility antigens. These histocompatibility differences do not stimulate consistent allograft reactions, and the histologic changes present in cardiac allografts transplanted between these strains were mild and variable 90 days posttransplantation. The animals in group 3 differed for a class I-like MHC antigen (RT1.E ${ }^{u}$ ) that has not been associated with evidence of allograft rejection in tissues other than skin (13). The histological appearance of the grafts exchanged between animals in this group did not differ from those seen in the control group and were consistent with the inability of this

TABLE 2. Histopathological lesions in donor hearts following allografting across defined genetic incompatibilities ${ }^{\circ}$

\begin{tabular}{cllrlllllll}
\hline Group & Donor & Recipient & $\mathrm{n}$ & VIP & PVF & MFD & MID & MFF & MIF & VAS \\
\hline 1 & DA & DA & 5 & 0 & 0 & 0 & 0 & 1.2 & 1.4 & 0 \\
2 & DA & DA.1I & 10 & 0.9 & 1.2 & 0 & 0.4 & 1.9 & 1.7 & 0 \\
3 & DA.1I & (DA $\times$ BN)F & 8 & 0 & 0 & 0 & 0 & 1.8 & 1.1 & 0 \\
4 & DA.1I & DA & 9 & 0 & 0.2 & 0.3 & 0.2 & 1.7 & 1.7 & 0 \\
5 & DA.1N & BN & 11 & 1.1 & 1.9 & 2.5 & 2.9 & 1.8 & 2.1 & 1.2 \\
6 & BN & DA.1N & 12 & 0.9 & 1.9 & 0.9 & 0.4 & 1.8 & 1.4 & 0.1 \\
7 & BN.1A & DA & 6 & 1.3 & 3.0 & 3.3 & 2.0 & 1.7 & 2.3 & 0.3
\end{tabular}

${ }^{a}$ Lesions are graded as described in the text, and the scores represent the mean grade for the animals within the group. VIP: vascular intimal proliferation; PVF: perivascular fibrosis; MFD: myocardial fibrosis, diffuse; MID: myocardial inflammation, diffuse; MFF: myocardial fibrosis, focal; MIF: myocardial inflammation, focal; VAS: vasculitis.

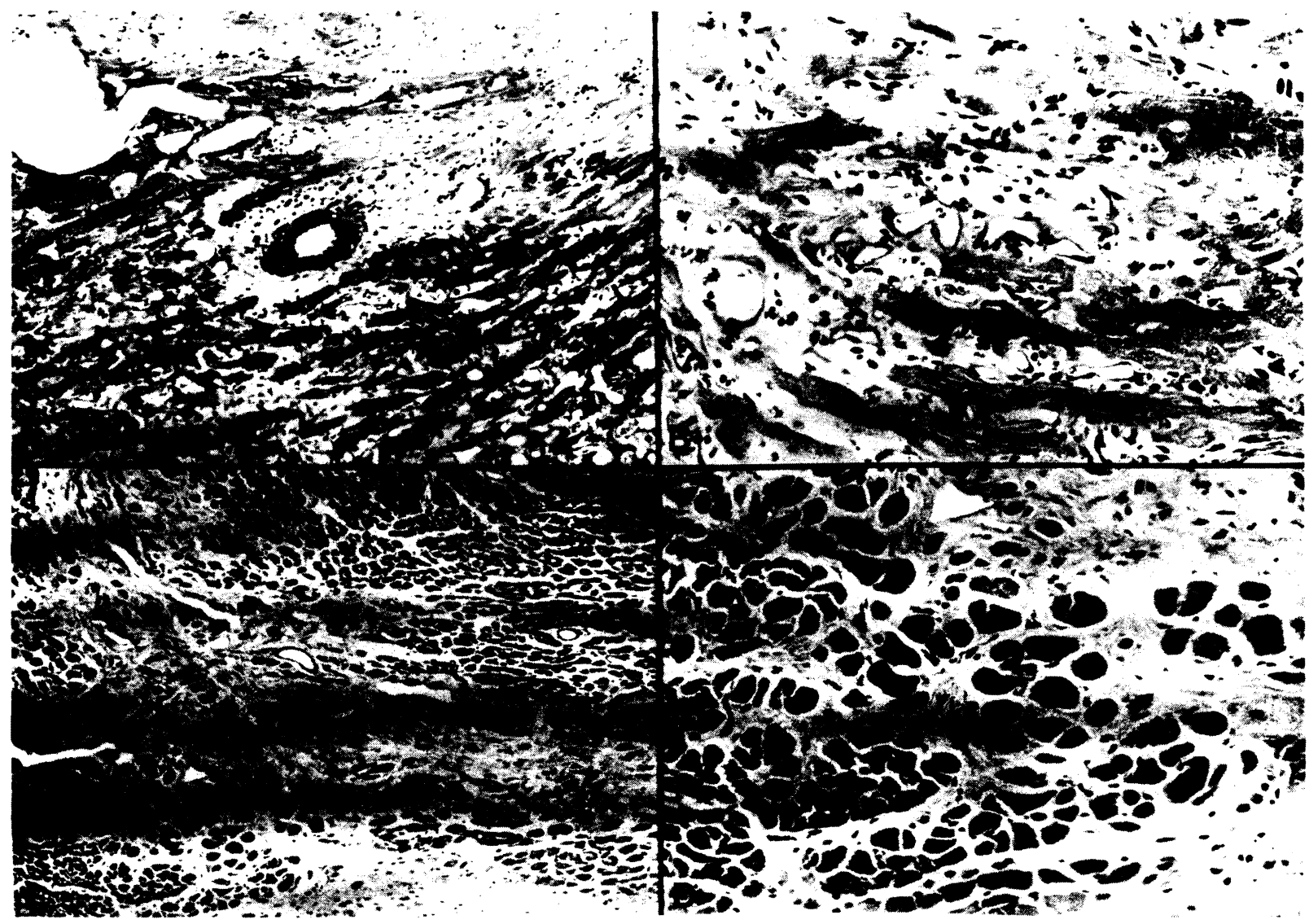

FIGURE 1. Histopathological changes in cardiac graft consisting of perivascular fibrosis, mild inflammatory cell infiltration, and fibrosis (A,B). Patchy areas of interstitial fibrosis with minimal numbers of inflammatory cells were also present (C,D). All sections were stained with hematoxylin and eosin $(\mathrm{A}: \times 300 ; \mathrm{B}: \times 750 ; \mathrm{C}: \times 300 ; \mathrm{D}: \times 750)$. 
Non-MHC incompatibilities: Three donor/recipient strain combinations differed for non-MHC histocompatibility antigens. The combination in which the non-MHC target antigens were contributed by the $\mathrm{BN}$ strain (group 6) had histologic changes that were similar in intensity and character to those described earlier for group 2 (RT1.A-incompatible). In the group 6 combination there was the appearance of trace amounts of interstitial fibrosis in 8 of 10 animals and a distinct mild-tomoderate level of perivascular proliferation in 9 animals.

The remaining two non-MHC strain combinations had lesions that were more advanced and intense. The animals in group 7 all exhibited a moderate-to-severe, diffuse fibrosis of the myocardium. The fibrosis was associated with a mild, diffuse mononuclear inflammatory infiltrate and moderate levels of perivascular fibrosis (Fig. 1). The inflammatory infiltrate consisted primarily of small lymphocytes and macrophages.

Of particular interest was the appearance of mild-to-moderate levels of intimal proliferation in several medium-to-large intramyocardial arteries (Fig. 2). The intimal lesions consisted of concentric fibrointimal cellular proliferation with luminal narrowing and partial occlusion of the vessels. The lesions were similar in extent and severity in groups 2 (RT1.A incompatible) and 5, 6 and 7 (non-MHC incompatible). The early lesions consisted of irregular endothelial proliferation, alterations in the internal elastic membrane, and infiltration of the vascular media with inflammatory cells, primarily macrophages (Figs. 3 and 4). The internal elastic membrane stained intensely with toluidine blue and was irregular in shape and discontinuous.

The lesions seen in group 5 consisted of intimal proliferation, perivascular fibrosis, and diffuse myocardial fibrotic lesions similar to those seen in group 7 . The character of these lesions was modified, however, by the appearance of a more severe inflammatory infiltrate of the myocardium. This inflammatory infiltrate was present in all 10 animals (moderate-to-severe in 8 ) and obscured the interstitial and perivascular fibrosis. Two animals in each of two groups (5 and 6) had focal areas of healed infarcts.

\section{DISCUSSION}

The appearance of arteriosclerosis in the coronary vessels of a cardiac allograft is a common and serious complication of heart transplantation (2). The vascular lesion tends to be diffuse, involving the more distal myocardial vessels and leading to the gradual occlusion of the vessels. In patients with cardiac allografts, significant coronary artery disease is present in approximately $50 \%$ of grafts that survive 5 years. The occlusion of coronary vessels by intimal proliferation is a common and sudden cause of death in graft recipients.

The pathogenesis of the coronary artery disease in cardiac allografts is not known. In experimental animals, the appearance of arteriosclerosis of myocardial vessels is associated with exchange of allogeneic grafts. Grafts exchanged between genetically identical (syngeneic) animals are generally free of vascular and myocardial lesions, demonstrating that the appearance of the lesions is not the result of the grafting procedure. When grafts are exchanged across allogeneic barriers, the arterial lesions appear, either caused by the recognition and rejection of the foreign graft or secondary to immunosuppression of the recipient to prevent graft rejection. In most studies, the prospective exchange of cardiac allografts has been conducted across allogeneic combinations in which loss of the graft

FIguRE 2. Concentric myointimal proliferation in medium-to-large myocardial arteries with narrowing of the lumen (A) and irregular focal areas of proliferation $(B, C)$. All sections were stained with hematoxylin and $\operatorname{eosin}(\mathrm{A}: \times 300 ; \mathrm{B}: \times 750 ; \mathrm{C}: \times 1200)$. 


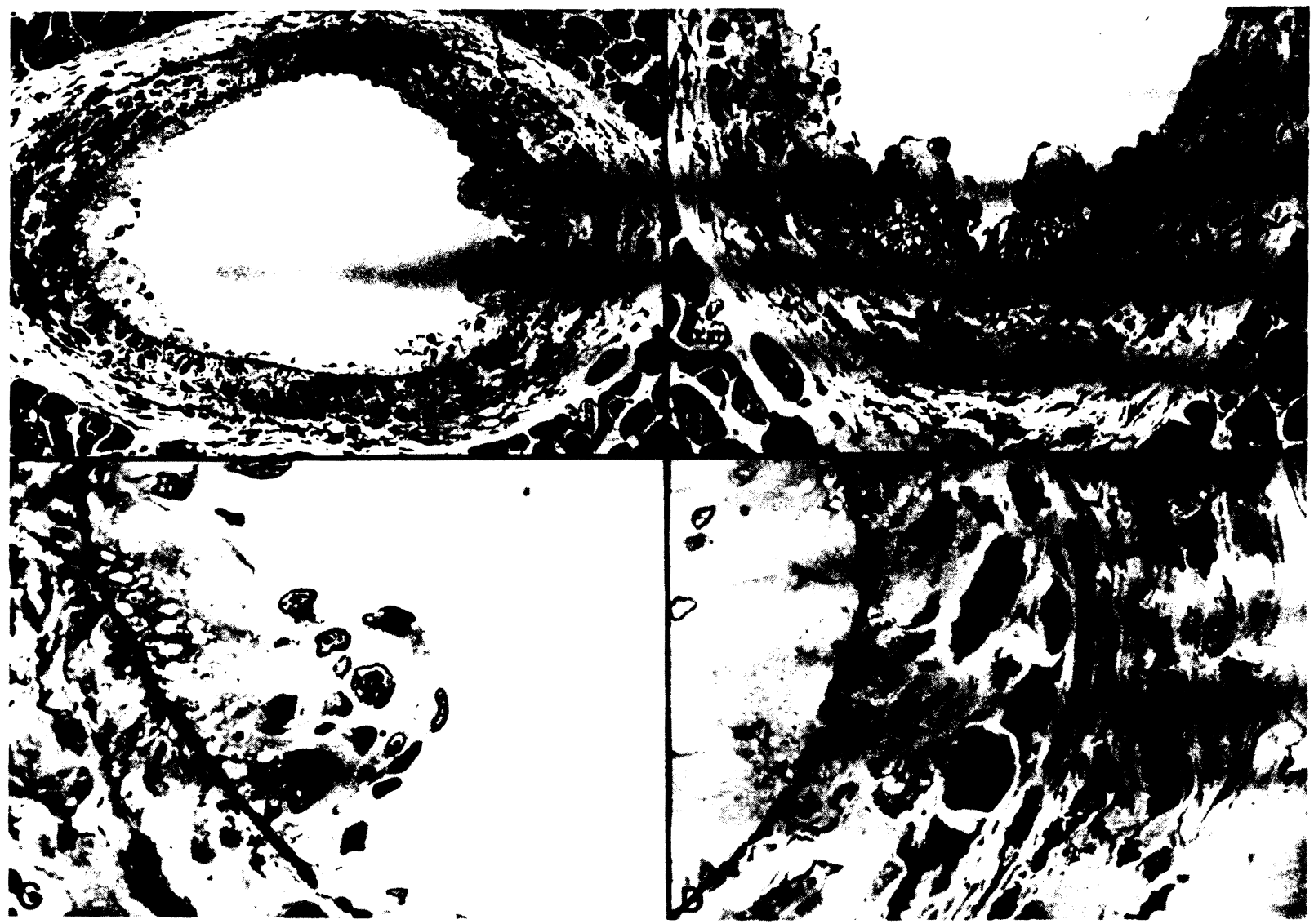

FIGURE 3. Early arterial lesions consisting of endothelial proliferation, disruption of prominently stained basement membrane (A,B,C), and infiltration of the vessel wall with macrophages (D). All sections stained with toluidine blue $(A: \times 750 ; B: \times 1200 ; C: \times 3000 ; D: \times 3000)$.

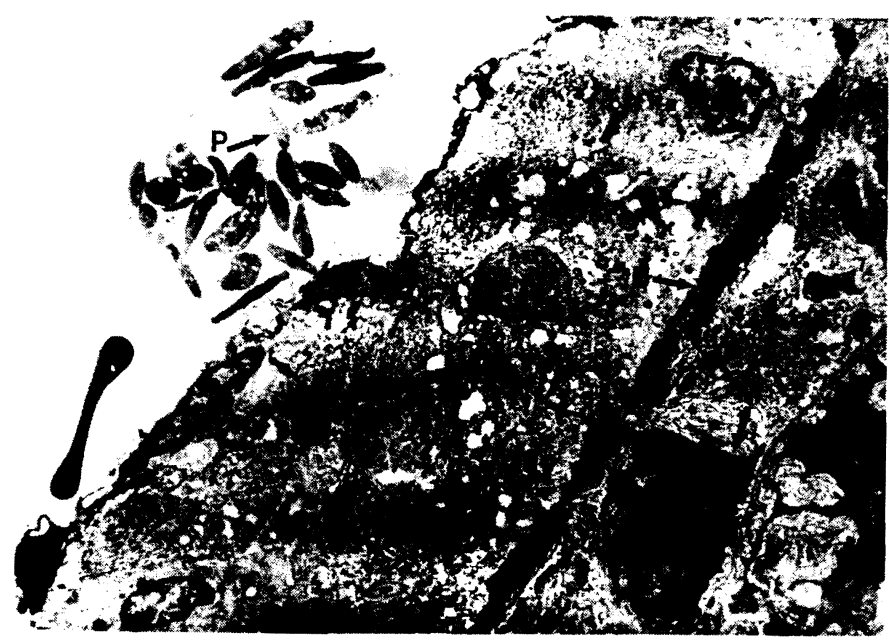

FIGURE 4. Electron photomicrograph of the vessel illustrated in Figure 3. The internal elastic membrane $(B)$ is irregular, electron dense and discontinuous. Macrophages (M) are present in the vessel wall and a small group of platelets $(P)$ is associated with the endothelial surface $(\times 7300$, moderately reduced).

from acute rejection occurs if the recipient is not treated with some form of immunosuppression. Under these conditions it has been difficult to establish the influence of immunosuppression on the development of the vascular lesions. This is particularly true for CsA, where arteriolar lesions, including intimal proliferation, have been associated with immunosuppression of renal allograft recipients $(9,10)$. At least one group of investigators have also suggested that immunosuppression, particularly with CsA, is capable of stimulating the atherosclerosis in cardiac grafts (17).

In the present study we have demonstrated that early arterial proliferative lesions, diffuse myocardial fibrosis, and perivascular fibrosis are histopathologic lesions associated with longterm (90-day) cardiac allografts. Grafting across histocompatibility differences that do not stimulate acute rejection allows for prolonged graft survival without immunosuppression and provides the opportunity for the emergence of the lesions in the donor heart. The type of histocompatibility difference does not appear to be important in determining the type of lesions present in the graft, as both MHC (class I) and non-MHC differences are capable of inducing one or more of the lesions described. Moreover, within the group of non-MHC incompatibilities, differences in the degree and character of the pathologic changes suggest that the most important feature of the disease process may be the strength of the allograft reaction. This interpretation of the data would be consistent with the results of previous experiments and suggests that the vascular changes represent a balance between the strength of the allograft reaction and sufficient time for the histopathological changes to appear.

The mechanism by which allogeneic differences stimulate the development of cardiac allograft lesions is unknown. In humans it has been suggested that a target for the allograft reaction is the vascular endothelium and that antibodies produced against endothelial cells (18) or against antigens shared 
by $B$ lymphocytes and the vascular endothelium (19) are reponsible for initiating the vascular lesions. In rats, a nonMHC endothelial antigen, Eag-1, is capable of stimulating the production of allospecific antibodies that bind to vascular endothelium, (20) and the antigen has been indirectly associated with renal rejection (21). The strains used in these studies differ for Eag-1, BN expressing the antigen and the DA and DA.1I strains being negative. The appearance of the vascular lesions in groups 2 and 5, where the Eag-1 antigen is not expressed, however, suggests that this antigen system is not the primary target of the allograft reaction. Although the Eag1 endothelial antigen system may not be the primary target of the allograft reaction, we have demonstrated that cardiac allorafts exchanged across various histocompatibility differences may stimulate active immune responses, including antibodies gainst both MHC and non-MHC antigens (11). These antibodies were directed against erythrocytic and lymphocytic antigens that may be expressed by the cells of the donor heart graft. Antibodies that react with antigens expressed specifically in the graft or against antigens shared with other tissues represent the most probable mechanism for initiating the vascular damage seen in these studies and is consistent with the histological changes observed in the affected vessels.

In summary, the exchange of heterotopic cardiac allografts between rat strains that display weak histocompatibility differences has been shown to stimulate the appearance of specific histopathological changes in the donor heart. These changes include diffuse fibrosis of the myocardium, perivascular fibrosis, and the appearance of arteriosclerosis. The arteriosclerosis consists of a concentric proliferation of myointimal cells in the distal branches of the coronary arteries and is associated with mild infiltration of the vessels with inflammatory cells and damage to the internal elastic membrane. The extent of the donor heart vascular lesions was correlated with the intensity of the allograft reaction and was not due to graft damage during the surgical procedure or to the administration of immunosuppressive agents.

\section{REFERENCES}

1. Griffith BP, Hardesty RL, Bahnson HT. Powerful but limited immunosuppression for cardiac transplantation with cyclosporins and low-dose steroid. J Thorac Cardiovasc Surg 1984; 87: 35 .

2. Barnhart GR, Pascoe EA, Mills AS, et al. Accelerated coronary arteriosclerosis in cardiac transplant recipients. In: Morris PJ, Tilney NL, eds. Transplantation reviews, vol 1. London: Grune \& Stratton, 1987: 31 .

3. Kosek JC, Hurley EJ, Lower RR. Histopathology of orthotopic canine cardiac homograft. Lab Invest 1968; 19: 97.
4. Laden AMK. The effect of treatment on the arterial lesions of rat and rabbit cardiac allografts. Transplantation 1972; 13: 281.

5. Alonso DR, Storek PK, Minick R. Studies on the pathogenesis of atherosclerosis induced in rabbit cardiac allografts by synergy of graft rejection and hypercholesterolemia. Amer J Pathol 1977; 87: 415.

6. Reemtsma K, Pierson RN III, Marboe CC, et al. Will atherosclerosis limit clinical xenografting? Transplant Proc 1987; 19: 108.

7. Laden AMK, Sinclair RA. Thickening of arterial intima in rat cardiac allografts. Amer J Pathol 1971; 63: 69.

8. Lurie KG, Billingham ME, Jamieson SW, Harrison DC, Reitz BA. Pathogenesis and prevention of graft arteriosclerosis in an experimental heart transplant model. Transplantation 1981; 31: 41.

9. Mihatsch MJ, Thiel J, Basler V, Landmann J, vonOverbeck J, Zollinger HU. Morphological patterns in cyclosporine-treated renal transplant recipients. Transplant Proc 1985; 17 (suppl 1): 101.

10. Sommer BG, Innes JT, Whitehurst RM, Sharma HM, Ferguson RM. Cyclosporine-associated renal arteriopathy resulting in loss of allograft function. Am J Surg 1985; 149: 756.

11. Katz SM, Liebert M, Gill TJ III, Kunz HW, Cramer DV, Guttmann $\mathrm{RD}$. The relative roles of MHC and non-MHC genes in heart and skin allograft survival. Transplantation 1983; 36: 96.

12. Katz SM, Cramer DV, Kunz HW, Gill TJ III. Effect of major histocompatibility complex genetic disparities on cardiac and skin graft rejection in the rat. Transplantation 1983; 36: 463.

13. Oaks MK, Cramer DV. The genetics of bone marrow transplantation in the rat. Transplantation 1985; 39: 69.

14. Tsuchimoto S, Mizuno K, Matsuno Y, et al. The effect of RT1 subregion differences on liver allograft survival in the rat. Transplantation 1985; 40: 218 .

15. Ono K, Lindsey ES. Improved technique of heart transplantation in rats. J Thorac Cardiovasc Surg 1969; 57: 225.

16. Cramer DV. Cardiac transplantation: immune mechanisms and alloantigens involved in graft rejection. CRC Crit Rev Immunol 1987; $7: 1$.

17. Muskett A, Burton NA, Eichwald EJ, et al. The effect of antiplatelet drugs on graft atherosclerosis in rat heterotopic cardiac allografts. Transplant Proc 1987; 19 (suppl 5): 74.

18. Cerilli J, Brasile L, Karmony A. Role of the vascular endothelial cell system in the etiology of atherosclerosis. Ann Surg 1985; 202: 329 .

19. Hess ML, Hastillo A, Mohanakumar T, et al. Accelerated atherosclerosis in cardiac transplantation: role of cytotoxic B-cell antibodies and hyperlipidemia. Circulation 1983; 68 (suppl 2): 94.

20. Blankert JJ, Van Es LA, Kunz HW, Cramer DV, Paul LC. Genetics of the antibody response to an endothelial transplantation antigen in the rat. Hum Immunol 1986; 15: 125.

21. Paul LC, Busch GJ, Paradysz JM, Carpenter CB. Definition, genetics, and possible significance of a newly defined endothelial antigen in the rat. Transplantation 1983; 36: 533.

Received 20 May 1988.

Accepted 11 July 1988. 